\title{
Pygmy dipole response of proton-rich argon nuclei in random-phase approximation and no-core shell model
}

\author{
C. Barbieri, ${ }^{1}$ E. Caurier ${ }^{2}$ K. Langanke, ${ }^{1,3}$ and G. Martínez-Pinedo ${ }^{1}$ \\ ${ }^{1}$ Gesellschaft für Schwerionenforschung Darmstadt, Planckstr. 1, D-64259 Darmstadt, Germany \\ ${ }^{2}$ Institut de Recherches Subatomiques, Université Louis Pasteur, F-67037 Strasbourg, France \\ ${ }^{3}$ Institut für Kernphysik, Technische Universität Darmstadt, Schlossgartenstr. 9, D-64289 Darmstadt, Germany
}

(Received 19 December 2007; published 19 February 2008)

\begin{abstract}
The occurrence of a pygmy dipole resonance in proton rich ${ }^{32,34} \mathrm{Ar}$ is studied using the unitary correlator operator method interaction $V_{\mathrm{UCOM}}$, based on Argonne V18. Predictions from the random-phase approximation (RPA) and the shell model in a no-core basis are compared. It is found that the inclusion of configuration mixing up to two-particles-two-holes broadens the pygmy strength slightly and reduces sensibly its strength, as compared to the RPA predictions. For ${ }^{32} \mathrm{Ar}$, a clear peak associated with a pygmy resonance is found. For ${ }^{34} \mathrm{Ar}$, the pygmy states are obtained close to the giant dipole resonance and mix with it.
\end{abstract}

DOI: 10.1103/PhysRevC.77.024304 PACS number(s): 21.10.Gv, 24.30.Gd, 24.10.Cn, 21.60.Cs

\section{INTRODUCTION}

Recent advances in experimental techniques for radioactive beams have fueled several studies of the properties of exotic nuclei, away from the line of $\beta$ stability. One of the most interesting results is the discovery of low-lying dipole strength in neutron-rich isotopes which is interpreted as a pygmy dipole resonance (PDR). This new excitation mode is then explained as the resonant oscillation of the weakly bound neutron skin against the isospin saturated proton-neutron core. Typically, one observes in nuclei with a neutron excess, i.e., $N>Z$, a concentration of electric dipole states close to the particle emission threshold. These carry a small fraction of the Thomas-Reiche-Kuhn (TRK) sum rule, which increases with the charge asymmetry of the nucleus. The PDR is also interesting because of its astrophysical implications. Although it carries only a small fraction of the total dipole strength, the occurrence of increased strength at the particle separation threshold can enhance radiative capture cross sections [1,2], which can have strong effects on the $r$-process nucleosynthesis and on the abundance distribution of elements. In addition, the thickness of the nuclear skin is directly related to the density dependence of the symmetry energy. Recently, data on the PDR resonance have been used to constrain models for the symmetry energy [3].

The onset of low-lying $E 1$ strength has been reported even in stable nuclei with moderate proton-neutron asymmetry such as ${ }^{44,48} \mathrm{Ca}$ and ${ }^{208} \mathrm{~Pb}$ [4-6]; see Ref. [7] for a review of high precision photon scattering experiments. For unstable nuclei with large neutron excess, a sizable fraction of low-lying E1 strength was observed in ${ }^{20,22} \mathrm{O}$ [8] and ${ }^{130,132} \mathrm{Sn}$ [9]. Several theoretical models have been employed to study the nature of this low-energy dipole strength. Recent works employed the Skyrme Hartree-Fock (HF) plus quasiparticle random-phase approximation (RPA) with phonon couplings $[10,11]$, the quasiparticle phonon model [12-14], and the relativistic quasiparticle RPA (RQRPA) $[15,16]$. In a recent work, the PDR was analyzed including effects of particlevibration coupling on top of the RPA approach [17]. The inclusion of low-lying phonons mainly correspond to consider explicit admixtures of two-particle-two-hole (2p2h) states and increase the fragmentation of the dipole distribution. In neutron-rich isotopes, it was found that this effect can generate a shift in the position of the PDR but otherwise does not noticeably change its characteristics.

On the proton-rich side, nuclei with an excess of protons over neutrons are found only for $Z \leqslant 50$. Due to the Coulomb repulsion, the proton drip line is much closer to the $\beta$ stability line, and proton skins are possible only for the lightest isotopes. For these elements, the multipole response is generally less collective. Although these facts seem to disfavor the existence of a proton PDR, a recent calculation suggested that this mode can actually be observed in medium-mass nuclei [16]. Using RQRPA calculations, low-lying pygmy states were obtained when approaching the proton drip line along the Ar isotopes and the $N=20$ isotones chains. However, no study of correlations beyond the RPA, such as in Ref. [17], has been reported to date for the proton PDR. In this work, we will consider the proton-rich isotopes ${ }^{32,34} \mathrm{Ar}$ and compare predictions for the dipole strength as obtained in the RPA with those from shell model (SM) studies in a no-core configuration space. These approaches include complementary correlation effects, since rather different portions of the Hilbert space are probed. Still, it is preferable to perform such a comparison based on the same Hamiltonian.

In the following, the unitary correlator operator method (UCOM) [18] will be employed to regularize the strong core of the realistic Argonne V18 potential [19]. The UCOM operator generates a unitary transformation within the many-body Hilbert space in which a weakly correlated wave function is mapped into one where strong short-range repulsion and tensor effects are explicitly manifested. If one applies this transformation to the Hamiltonian, rather than the wave function, it finds an expansion of the effective interaction into many-body terms. The $V_{\mathrm{UCOM}}$ force is obtained by truncating the expansion at the two-body level. The result is an effective force that tames the strong short-range and tensor components of the original force and is therefore applicable to smaller basis sets. The subtle cancellation between large nuclear and 
kinetic contributions to the total energy is also accounted for in this expansion. Since the correlator operator is chosen in such a way that nucleon-nucleon phase shifts are preserved, $V_{\mathrm{UCOM}}$ can also be regarded as a realistic two-nucleon force on its own that is applicable, however, only to medium- and low-energy processes. Therefore, the UCOM method provides an interaction independent of the employed model space and can be applied meaningfully to both the RPA and the SM methods. At the same time, it includes the correlation effects proper for modern high precision realistic interactions.

Details of the formalism employed in the calculations are discussed in Sec. II. The RPA and the shell model results are compared in Sec. III. Before presenting these results, we will discuss the dependence of our calculations on the parameters of the oscillator space and use the Hartree-Fock (HF) approach to construct a basis to be used in the shell model studies, in Sec. III A. Conclusions are drawn in Sec. IV.

\section{FORMALISM}

Consistent RPA calculations were performed to guarantee the exact separation of the spurious center-of-mass motion. This means that our single-particle basis was obtained by solving the HF problem, and the same interaction was used for both HF and RPA. To do this, one must take some care in treating the $d_{3 / 2}$ orbit which contains only two protons in the mean-field picture. To maintain consistency and to ensure the separation of spurious states, one must (1) force uniform occupation for protons in this orbit when solving the HF equations, (2) account for its partial occupation when solving the RPA problem, and (3) allow for excitations of protons both from the $d_{3 / 2}$ to higher orbits and from lower orbits into the (half empty) $d_{3 / 2}$ level. This was done by considering the latter orbit both as a particle and as a hole state, with the respective depletion or occupation probabilities. Indicating the HF basis with $a \equiv n_{a} l_{a} j_{a} \tau_{a}$ ( $\tau$ is the isospin), the RPA eigenvalue equations for the amplitudes $X_{a b}^{J}$ and $Y_{a b}^{J}$ are

$$
\left(\begin{array}{cc}
\mathbf{A}^{\mathbf{J}} & \mathbf{B}^{\mathbf{J}} \\
\mathbf{B}^{\mathbf{J}^{*}} & \mathbf{A}^{\mathbf{J}^{*}}
\end{array}\right)\left(\begin{array}{l}
X^{J, v} \\
Y^{J, v}
\end{array}\right)=\omega_{v}\left(\begin{array}{cc}
1 & 0 \\
0 & -1
\end{array}\right)\left(\begin{array}{l}
X^{J, v} \\
Y^{J, v}
\end{array}\right),
$$

where

$$
\begin{aligned}
A_{a b, c d}^{J} & =n_{a} \bar{n}_{b} H_{a b, c d}^{J} n_{c} \bar{n}_{d}+\delta_{a c} \delta_{b d}\left(\varepsilon_{a}-\varepsilon_{b}\right), \\
B_{a b, c d}^{J} & =n_{a} \bar{n}_{b} H_{a b, d c}^{J} \bar{n}_{d} n_{c},
\end{aligned}
$$

where $J$ is the angular momentum of the excited state, $\varepsilon_{a}$ is the HF single-particle energy of orbit $a$, and $H_{a b, c d}^{J}$ the Pandya transform of the residual two-body interaction. The numbers $\left(n_{a}\right)^{2}$ define the occupation of orbit $a$ in the HF wave function, while $\left(\bar{n}_{a}\right)^{2}$ measures the unoccupied space. Hence, for the Ar isotopes, we have

$$
\left(n_{a}\right)^{2}=1-\left(\bar{n}_{a}\right)^{2}= \begin{cases}1 & \text { for } a \text { fully occupied } \\ 0 & \text { for } a \text { empty } \\ 1 / 2 & \text { for } a=\pi 0 d_{3 / 2}\end{cases}
$$

In Eqs. (1) and (3), the products $n_{a} \bar{n}_{b}$ are restricted to $1 \mathrm{p} 1 \mathrm{~h}$ configurations only, except for the partially occupied orbit. It must be stressed that the above prescription arises naturally when deriving the HF+RPA scheme from propagator (or Green's function) theory [20,21]: following Baym and Kadanoff [22,23], conserving RPA equations are derived consistently from the HF self-energy and propagator. The HF propagator, however, contains both particle and hole poles for each partially occupied orbit, such as the $d_{3 / 2}$ in our case. We checked that neglecting proton excitations from lower orbits to the $d_{3 / 2}$ level no longer allows for the exact separation of the spurious center-of-mass mode (although the breaking is small and has negligible effects on the remaining $E 1$ strength). The RPA results reported in this paper are obtained in the fully conserving approach. In all cases, we consider the isovector dipole operator

$$
\hat{Q}_{1 m}^{T=1}=\frac{N}{N+Z} \sum_{p=1}^{Z} r_{p} Y_{1 m}-\frac{Z}{N+Z} \sum_{n=1}^{N} r_{n} Y_{1 m},
$$

which is corrected for the center-of-mass displacement. In all figures below, the calculated $B(E 1)$ strength to each final state $f$,

$$
B_{f i}(E 1)=\frac{1}{2 J_{i}+1}\langle f\|\hat{Q}\| i\rangle,
$$

was folded with a Lorentzian of width $\Gamma=1 \mathrm{MeV}$,

$$
R\left(E_{x}\right)=\sum_{f} B_{f i}(E 1) \frac{\Gamma / 2 \pi}{\left(E_{x}-\omega_{f}\right)^{2}+\Gamma^{2} / 4} .
$$

However, the reported integral quantities are always calculated by considering the full strength of each peak concentrated at the respective eigenvalue.

In all calculations, we employ the $V_{\mathrm{UCOM}}$ interaction and subtract the kinetic energy of the center-of-mass motion. The intrinsic Hamiltonian is then written in the form of a two-body interaction

$$
H_{\mathrm{int}}=T+V-T_{\mathrm{c} . \mathrm{m} .}=\sum_{i<j}\left\{\frac{\left(\mathbf{p}_{i}-\mathbf{p}_{j}\right)^{2}}{2 A m_{N}}+V_{i, j}\right\} .
$$

In all cases, a UCOM operator was used which corresponds to a correlation volume of $I_{\vartheta}=0.09 \mathrm{fm}^{3}$ for the tensor force. This correlator was tuned to reproduce the binding energies of ${ }^{4} \mathrm{He}$ and ${ }^{3} \mathrm{H}$ without the need for additional three-nucleon forces [24]. Subsequent calculations suggested that binding energies are reproduced, in perturbation theory, throughout the nuclear chart without additional corrections to this UCOM operator $[25,26]$.

A harmonic oscillator basis with length $b_{\mathrm{HO}}=1.8 \mathrm{fm}$ was used for all the RPA calculations, except for ${ }^{32} \mathrm{Ar}$ when indicated. This basis was truncated according to the number of major shells $2 n+l \leqslant N_{\max }$ (with $N_{\max }=0,1, \ldots$ ). Calculations based on $V_{\mathrm{UCOM}}$ generally converge in model spaces of 10-20 shells if a harmonic oscillator basis is used. Standard applications of the no-core shell model (NCSM) [27] are based on using harmonic oscillator bases and truncating the model space in terms of the maximum number of cross shell excitations. The reason for doing so is to allow for an exact separation of the center-of-mass motion. As it will be shown in Sec. III A, a very large number of oscillator functions would be required for converging the excitation energy of the pygmy state. Obviously, this is beyond the 
capability of present-day computers, and one needs to resort to a more realistic single-particle basis. Below, we will expand the Hamiltonian, Eq. (7), over the lowest HF orbits that are obtained while solving the corresponding HF+RPA problem. Since we will only consider model spaces in which all nucleons are active, we refer to this scheme as NCSM. The secular problem was diagonalized using the shell model code ANTOINE $[28,29]$. The dipole response was derived as usual by first applying the operator $\hat{Q}$ to the $\mathrm{J}^{\pi}=0^{+}$ground state of ${ }^{32,34} \mathrm{Ar}$, and then using the resulting $1^{-}$wave function as a pivot for successive Lanczos iterations.

\section{RESULTS}

\section{A. Dependence on the model space}

Figure 1 shows the dipole strength distribution, Eq. (6), obtained with RPA for different model spaces and oscillator lengths. In all cases, one can identify a peak on the low-energy tail of the isovector giant dipole resonance (IVGDR) which receives strength almost exclusively from proton excitations, that is, from the first term on the right-hand side of Eq. (4). As it will be discussed below, this represents the PDR. Unfortunately, very large configuration spaces are needed to converge the pygmy peak at low energies. This behavior is an artifact of employing a harmonic oscillator basis, which does not properly describe loosely bound states. The dominant contributions to the PDR come from the collective excitation of protons to states just above the continuum threshold. Hence,

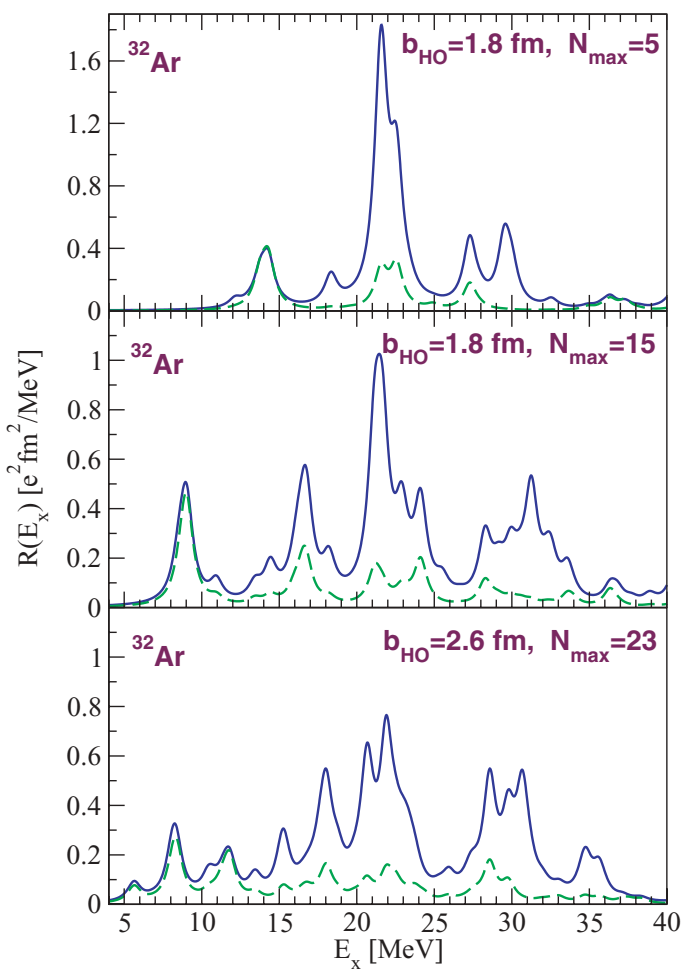

FIG. 1. (Color online) Isovector dipole strength of ${ }^{32} \mathrm{Ar}$ obtained with HF+RPA, for different sizes of the model space $\left(N_{\max }\right)$ and harmonic oscillator lengths $\left(b_{\mathrm{HO}}\right)$. The dashed lines represent the contribution from protons only [first term in Eq. (4)].
TABLE I. Total $E 1$ strength (in $e^{2} \mathrm{fm}^{2}$ ) for ${ }^{32} \mathrm{Ar}$ obtained with $\mathrm{HF}+\mathrm{RPA}$ for energies below 12 and $40 \mathrm{MeV}$. The centroids of these distributions are also reported (in $\mathrm{MeV}$ ).

\begin{tabular}{lccccc}
\hline \hline & \multicolumn{2}{c}{$E_{x} \leqslant 12 \mathrm{MeV}$} & & \multicolumn{2}{c}{$E_{x} \leqslant 40 \mathrm{MeV}$} \\
\cline { 2 - 3 } \cline { 5 - 6 } Basis & $\bar{E}$ & $\sum B(E 1)$ & & $\bar{E}$ & $\sum B(E 1)$ \\
\hline$b_{\mathrm{HO}}=1.8 \mathrm{fm}:$ & & & & \\
$N_{\max }=5$ & - & - & & 23.06 & 7.13 \\
$N_{\max }=9$ & 10.71 & 1.03 & & 22.69 & 8.01 \\
$N_{\max }=11$ & 10.12 & 1.08 & & 22.14 & 7.86 \\
$N_{\max }=15$ & 9.15 & 1.01 & & 22.26 & 8.04 \\
$N_{\max }=19$ & 8.72 & 0.97 & & 22.14 & 8.05 \\
$N_{\max }=23$ & 8.51 & 0.86 & & 22.17 & 8.09 \\
$b_{\mathrm{HO}}=2.6 \mathrm{fm}:$ & & & & \\
$N_{\max }=15$ & 9.03 & 1.13 & & 21.99 & 8.27 \\
$N_{\max }=19$ & 8.59 & 0.93 & & 22.22 & 8.14 \\
$N_{\max }=23$ & 9.28 & 1.14 & & 22.12 & 8.09 \\
\hline \hline
\end{tabular}

rather diffuse wave functions may be needed to properly describe this strength. This implies large harmonic oscillator bases. An inspection of the HF single-particle energies of protons show that the orbits in the $s d$ shell are well converged already for $N_{\max }=5$, while the $p f$ orbits are found above the separation energy and keep changing when increasing the model space.

To assess the sensitivity of our results to the choice of the model space, RPA calculations were performed up to $N_{\max }=23$ (24 major oscillator shells). For the largest model spaces, the HF ground state properties are independent of the oscillator length used. Hence, we have also performed calculations with a larger oscillator length, $b_{\mathrm{HO}}=2.6 \mathrm{fm}$, to facilitate the description of states in the continuum. The centroid and total strength of the resulting $E 1$ distributions are reported in Table I for energies up to 12 and $40 \mathrm{MeV}$. It must be stressed that the pygmy behavior is reproduced by RPA theory even for the smallest model spaces considered here, and its existence is therefore a stable prediction. However, no strength is obtained in the low-energy region when a small number of oscillator functions is employed. Increasing the space, the pygmy peak is lowered, and it eventually stabilizes at around $9 \mathrm{MeV}$. Table I shows that 16 major oscillator shells are required to converge the centroid of the IVGDR and the summed strength in the region below $12 \mathrm{MeV}$. Increasing the space further, small variations of the results still occur which are a result of discretizing the continuum with an increasing number of single-particle orbits. The limit of an infinite space would be reached by solving directly the continuum RPA equations. However, we do not expect that this would lead to sensible deviations for the integral quantities of Table I.

Although very large oscillator bases are needed to generate the proper HF wave functions, only the orbits near the Fermi surface are actually relevant for constructing the pygmy resonance. This is depicted in Fig. 2, in which the distribution obtained in the full $N_{\max }=15$ model space is compared to the one obtained using the same HF basis but restricted to the six lowest shells. Solving the RPA equations in this basis is sufficient to reproduce the original results up to an excitation 


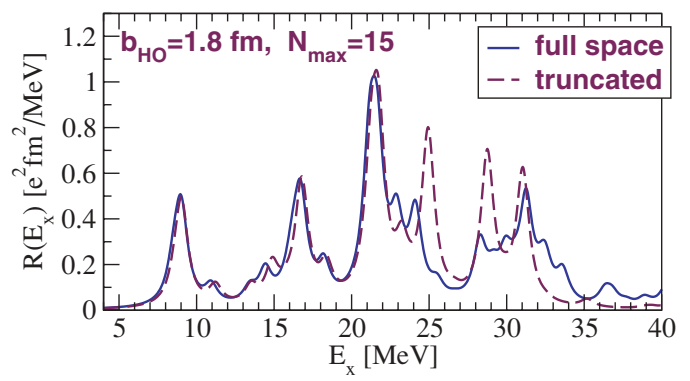

FIG. 2. (Color online) Dependence of the HF+RPA strength distribution on the truncation of the single-particle basis. The same $\mathrm{HF}$ basis is employed in both calculations, and was derived in a $N_{\max }=15$ harmonic oscillator space. Full line: all the HF states are used to solve the RPA equations. Dashed line: only the six lowest HF shells were retained.

energy of $\approx 23 \mathrm{MeV}$. The summed strength and centroid below $40 \mathrm{MeV}$, obtained in this way, are $22.0 \mathrm{MeV}$ and $7.97 e^{2} \mathrm{fm}^{2}$, which underestimates the total strength by only a percent due to truncation. Thus these quantities remain largely unaffected. The truncation of the HF basis reproduces the pygmy results obtained with the larger harmonic oscillator space. It is also small enough to allow no-core shell model calculations, which are presented in Sec. III C.

We note that not only the matrix elements of the Hamiltonian, Eq. (7), were expanded in the new HF basis, but also the matrix elements of the dipole operator $\hat{Q}$. Employing the correct one-body matrix elements of this operator was found to be crucial to reproducing the correct transition strengths.

\section{B. RPA results for ${ }^{32} \mathrm{Ar}$}

From Table I we deduce that with the present choice of the correlator $\left(\mathrm{I}_{\vartheta}=0.09 \mathrm{fm}^{3}\right)$ and at the RPA level, the $V_{\mathrm{UCOM}}$ interaction predicts an isovector dipole strength of $8.15 \pm$ $0.10 e^{2} \mathrm{fm}^{2}$ within the first $40 \mathrm{MeV}$ of excitation energy. The centroid of this distribution is $E_{\mathrm{IVGDR}}=22.15 \pm 0.15 \mathrm{MeV}$. This is close to the empirical estimate $E_{\text {peak }}=31.2 / A^{1 / 3}+$ $20.6 / A^{1 / 6}=21.4 \mathrm{MeV}$ [30]. However, one should keep in mind that this formula was derived from data on stable nuclei with masses $A>50$, and it is just indicative in this case. In the energy region up to $40 \mathrm{MeV}$, the RPA calculation exhausts about $153 \%$ of the TRK sum rule. This enhancement is expected for realistic interactions, and it can be traced to the strong tensor component of the $V_{\mathrm{UCOM}}$ force [31]. A similar enhancement has also been reported applying $V_{\mathrm{UCOM}}$ to ${ }^{4} \mathrm{He}$ [32]. Examples for the transition densities to states around $20 \mathrm{MeV}$ are shown in Fig. 3. These describe an out-of-phase oscillation of protons and neutrons and confirm the IVGDR nature of these excitations.

More interesting is the pygmy peak at the lower end of the GDR tail. As already noted, this receives $E 1$ strength almost exclusively from protons - the first term on the right-hand side of Eq. (4) - as shown by the dashed lines in Fig. 1. The transition densities for the RPA states at 8.45, 8.60, and $10.94 \mathrm{MeV}$ are reported in Fig. 3 and show the typical behavior of the PDR: protons and neutrons move in phase
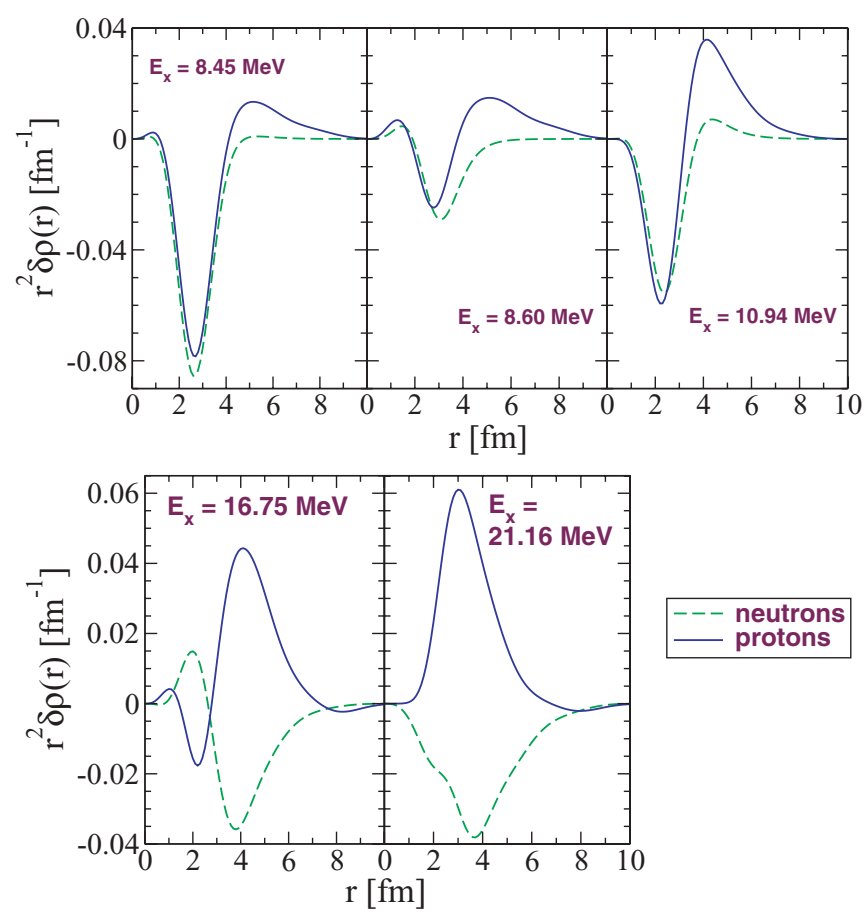

FIG. 3. (Color online) Proton and neutron transition densities for ${ }^{32}$ Ar obtained from RPA theory $\left(N_{\max }=15\right)$. Three states associated with the proton pygmy resonance (at $8.45,8.60$, and $10.94 \mathrm{MeV}$ ) are compared to the IVGDR eigenstates at 16.75 and $21.16 \mathrm{MeV}$.

in the nuclear interior, while only protons are excited at the surface and extend outside the nuclear core. The total strength found below $12 \mathrm{MeV}$ of excitation energy is $1.0 \pm 0.15 e^{2} \mathrm{fm}^{2}$, for $N_{\max } \geqslant 15$. No strength is seen in this region if only six oscillator shells are retained. In this case, the PDR is obtained at larger energies, but it carries a similar strength $\left(0.84 e^{2} \mathrm{fm}^{2}\right.$ up to $E_{x}=15 \mathrm{MeV}$ ).

\section{NCSM results for ${ }^{32} \mathrm{Ar}$}

No-core shell model calculations were done in order to investigate the effect of correlations beyond RPA on the dipole distribution. As indicated in Fig. 1, an oscillator basis $N_{\max }=15$, corresponding to a $13 \hbar \omega$ model space, would be required to converge the energy of the PDR. The number of active single-particle orbits can be reduced by employing the truncated HF basis discussed in Sec. III A. The proton $\mathrm{HF}$ orbits were used to expand the $V_{\mathrm{UCOM}}$ interaction and the dipole operator. The explicit configurations employed were $\left(0 s_{1 / 2} 0 p_{3 / 2} 0 p_{1 / 2} 0 d_{5 / 2}\right)^{14-n_{v}}\left(1 s_{1 / 2} 0 d_{3 / 2} 0 f_{7 / 2} \ldots 0 h_{11 / 2} 0 h_{9 / 2}\right)^{n_{v}}$ for neutrons and $\left(0 s_{1 / 2} 0 p_{3 / 2} 0 p_{1 / 2} 0 d_{5 / 2} 1 s_{1 / 2} 0 d_{3 / 2}\right)^{18-n_{\pi}}$ $\left(0 f_{7 / 2} 0 f_{5 / 2} 1 p_{3 / 2} \ldots 0 h_{11 / 2} 0 h_{9 / 2}\right)^{n_{\pi}}$ for protons. The model space was truncated in terms of the total number of particle-hole excitations $\left(n_{v}+n_{\pi} \leqslant n\right)$. This maintains all the particles active and allows for excitations from the core. The $0^{+}$ground state was first derived using an $n$-particle- $n$-hole $(n \mathrm{p} n \mathrm{~h})$ model space. Once this is done, the dipole operator, Eq. (4), connects this to states in a space containing up to $(n+1) \mathrm{p}-(n+1) \mathrm{h}$. By performing Lanczos diagonalization of the $1^{-}$final states in the larger configuration space, one 




FIG. 4. (Color online) Top panels: isovector spectral strength obtained with the shell model for dipole excitations to the $1 \mathrm{p} 1 \mathrm{~h}$ and $2 \mathrm{p} 2 \mathrm{~h}$ configuration spaces. The first six HF orbits were included in the basis. Bottom panel: $1 \hbar \omega$ result, including excitations up to the pf shell only.

is guaranteed to exhaust the total dipole strength. Note that for a $0 \mathrm{p} 0 \mathrm{~h}$ space, the neutrons are constrained to the lowest $\mathrm{HF}$ orbits while some mixing is possible for protons, which can be excited from the lower shells into the half-empty $d_{3 / 2}$ orbit. This already leads to a configuration space beyond the corresponding RPA theory.

The upper panels of Fig. 4 show the strength distributions obtained from the $0 \mathrm{p} 0 \mathrm{~h}-1 \mathrm{p} 1 \mathrm{~h}$ and $1 \mathrm{p} 1 \mathrm{~h}-2 \mathrm{p} 2 \mathrm{~h}$ configuration spaces. The same structure of an IVGDR resonance and pygmy peak is found, as for the RPA (Fig. 2), but both the IVGDR distribution and PDR are broadened by shell model correlations. For the $2 \mathrm{p} 2 \mathrm{~h}$ model space, the IVGDR is centered at $21.66 \mathrm{MeV}$. Integrating up to $40 \mathrm{MeV}$ excitation energy, one finds that the total strength is lowered by configuration mixing and exhausts $137 \%$ of the TRK sum rule. The response in the pygmy region is compared with the RPA approximation in Table II. Shell model correlations do not significantly alter the position of the PDR. On the other hand, its strength is reduced by a factor of 2 .

The configuration spaces used above restrict most of the neutrons in the $d_{5 / 2}$ orbit and below. One may question whether neutron correlations in the $s d$ shell affect the properties of the PDR. To check this, we performed a NCSM calculation in the $1 \hbar \omega$ model space, that is, by opening the $s d$ shell and allowing for one excitation across major shells. The strength distribution, plotted in the lower panel of Fig. 4, shows no qualitative distortion of the pygmy peak. Hence,
TABLE II. Total dipole strength $\left(e^{2} \mathrm{fm}^{2}\right)$ and its centroid $(\mathrm{MeV})$ in the region of the pygmy resonance, as obtained in RPA and shell model with $1 \mathrm{p} 1 \mathrm{~h}$ and $2 \mathrm{p} 2 \mathrm{~h}$ configurations. The dipole distribution was integrated up to $12 \mathrm{MeV}$ of excitation energy for ${ }^{32} \mathrm{Ar}$ and $14 \mathrm{MeV}$ for ${ }^{34} \mathrm{Ar}$.

\begin{tabular}{lcclcc}
\hline \hline & \multicolumn{2}{c}{${ }^{32} \mathrm{Ar}$} & & \multicolumn{2}{c}{${ }^{34} \mathrm{Ar}$} \\
\cline { 2 - 3 } \cline { 5 - 6 } & $\bar{E}_{\mathrm{pyg}}$ & $\sum B_{\mathrm{pyg}}(E 1)$ & & $\bar{E}_{\mathrm{pyg}}$ & $\sum B_{\mathrm{pyg}}(E 1)$ \\
\hline RPA & 9.15 & 1.0 & & 12.7 & 0.8 \\
$1 \mathrm{p} 1 \mathrm{~h}$ & 9.66 & 0.44 & & 12.8 & 0.65 \\
$2 \mathrm{p} 2 \mathrm{~h}$ & 8.95 & 0.49 & & 11.6 & 0.62 \\
\hline \hline
\end{tabular}

the approximation made restricting neutrons in the $0 \mathrm{p} 0 \mathrm{~h}$ configuration should not noticeably affect our conclusions. The PDR strength is also reduced here to about $60 \%$ of the RPA value. However, at $1 \hbar \omega$, only excitations to the $p f$ shell are possible. Since this corresponds to a more severe truncation of the HF basis, we do not attempt to extract quantitative information on the PDR from this calculation.

\section{Results for ${ }^{34} \mathrm{Ar}$}

A similar analysis was carried out for ${ }^{34} \mathrm{Ar}$. The strength predicted from RPA theory is depicted in Fig. 5 for $N_{\max }=$ 15. The centroid of the dipole distribution in the interval $[0$, 40] $\mathrm{MeV}$ is obtained as $23.3 \mathrm{MeV}$. Summing this strength within the same interval gives $8.69 e^{2} \mathrm{fm}^{2}$, which corresponds to $161 \%$ of the TRK sum rule. These predictions do not vary

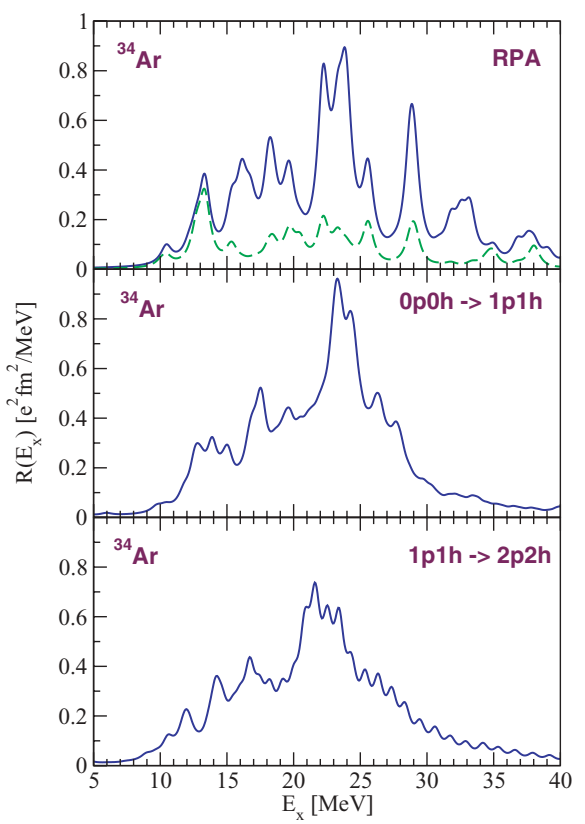

FIG. 5. (Color online) Isovector spectral strength for ${ }^{34} \mathrm{Ar}$ obtained with HF+RPA (top) and the shell model up to $1 \mathrm{p} 1 \mathrm{~h}$ and $2 \mathrm{p} 2 \mathrm{~h}$ (center and bottom). The full $N_{\max }=15$ harmonic oscillator space was employed in the RPA calculations. The same HF basis derived for the RPA calculation is used for the shell model, but truncated to the lowest six shells. 


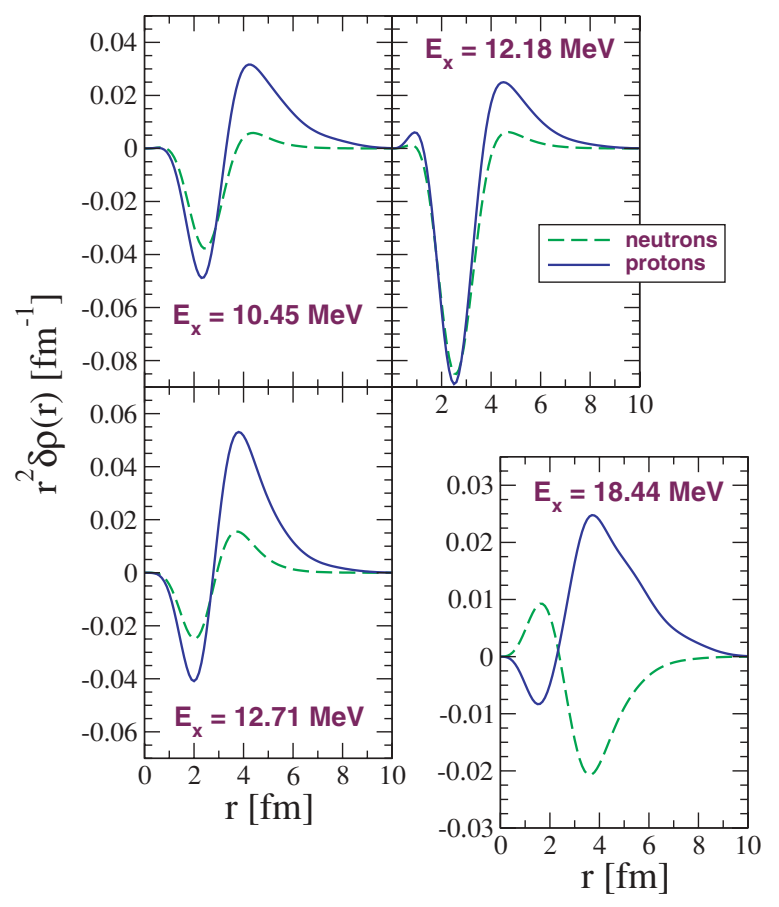

FIG. 6. (Color online) RPA transition densities for the excitations to three pygmy states of ${ }^{34} \mathrm{Ar}$ (at $10.45,12.18$, and $12.71 \mathrm{MeV}$ ) and to an IVGDR state at $18.44 \mathrm{MeV}$.

by increasing the model space and the oscillator parameter. A similar analysis as that done for ${ }^{32} \mathrm{Ar}$ suggests an uncertainty of $\pm 0.8 \mathrm{MeV}$ and $\pm 0.2 e^{2}, \mathrm{fm}^{2}$ for these quantities.

A pygmy resonance is also found for this isotope but closer to the centroid of the IVGDR. The dashed line in Fig. 5 shows that a sizable proton contribution to the dipole response strength is found up to about $14 \mathrm{MeV}$. An analysis of the transition densities (Fig. 6) confirms that these states have the character of a pygmy mode. Instead, the RPA eigenstates found just a few MeVs above contribute to the lower tail of the IVGDR. To estimate the total strength associated with the pygmy resonance, we integrated $R\left(E_{x}\right)$ up to $14 \mathrm{MeV}$ and found $0.80 e^{2} \mathrm{fm}^{2}$.

No-core shell model calculations were performed in a model space analogous to the ${ }^{32} \mathrm{Ar}$ case, obtained by solving the HF equation for ${ }^{34} \mathrm{Ar}$ in the same harmonic oscillator space as used for RPA. This basis was then truncated to six major shells and, at the 0p0h level, neutrons were constrained in the $d_{5 / 2}$ and $s_{1 / 2}$ orbits. Also in this case, we found a slight reduction of the total strength with respect to the RPA result, yielding $8.21 e^{2} \mathrm{fm}^{2}$ for the $1 \mathrm{p} 1 \mathrm{~h}-2 \mathrm{p} 2 \mathrm{~h}$ configurations space. In this case, the centroid of the IVGDR was lowered to $22.0 \mathrm{MeV}$, and the energy weighted sum rule reduces to $143 \%$ of the TRK value, in the first $40 \mathrm{MeV}$. The strength resulting from the shell model calculation is plotted in the lower panels of Fig. 5 and shows a broad distribution of the $E 1$ strength. Since for this nucleus the pygmy peak is obtained close to the giant resonance, the two are strongly mixed, and one finds just a long tail spreading in the low-energy region. From the figure, it is not possible to clearly identify a separate peak corresponding to the pygmy resonance. Table II lists the summed strength below $14 \mathrm{MeV}$, where one expects to find most of the pygmy states. Contrary to the ${ }^{32} \mathrm{Ar}$ case, the effects of configuration mixing on the low-energy dipole strength is moderate. A reduction of about $20 \%$ with respect to the RPA result was found.

\section{CONCLUSIONS AND DISCUSSION}

The properties of the pygmy dipole resonance in the protonrich isotopes ${ }^{32} \mathrm{Ar}$ and ${ }^{34} \mathrm{Ar}$ were investigated by comparing the predictions of the RPA and the shell model in a no-core configuration space. The $V_{\mathrm{UCOM}}$ interaction was employed in all cases as a low-energy realistic Hamiltonian. In this approach, the nuclear force is tamed to account for the effects on short-range correlations and can be directly applied in large-scale RPA and shell model calculations. In general a large number of harmonic oscillator shells is still required to converge the distribution of dipole strength in the low-energy region. However, it was seen that the number of active orbitals can be significantly reduced using the HF single-particle basis. Therefore, comparisons could be done employing the same Hartree-Fock basis for the RPA and shell model.

For ${ }^{32} \mathrm{Ar}$, enhanced low-lying strength was found at energies up to $12 \mathrm{MeV}$, which could be identified as proton PDR. The corresponding strength distribution is peaked at about $9 \mathrm{MeV}$, considerably above the experimental proton separation energy of $2.4 \mathrm{MeV}$ [33]. This should not come as a surprise, since oscillations of the proton skin need to overcome the Coulomb barrier. The RPA approximation predicts about $1.0 e^{2} \mathrm{fm}^{2}$ in this energy region. Additional correlations, as accounted for in shell model studies, reduce this strength. Furthermore the pygmy peak is slightly broadened due to a larger number of configurations. By explicitly including up to $2 \mathrm{p} 2 \mathrm{~h}$ states, the pygmy strength is halved to $0.49 e^{2} \mathrm{fm}^{2}$. The results obtained here predict a well-defined pygmy peak separated from the IVGDR, in accordance with the previous RQRPA calculations of Paar et al. [16]. Excitations associated with a PDR were also found for ${ }^{34} \mathrm{Ar}$, which has only two more protons than neutrons. The present calculations place its strength at energies up to $14 \mathrm{MeV}$ and close to the IVGDR. The total strength found up to this energy is also reduced by shell model calculations, as compared to RPA. However, the mixing with the nearby giant resonance states leads to a rather uniform response without a well-defined pygmy peak.

We note that the neutron PDR observed in larger nuclei is typically found below $10 \mathrm{MeV}$, and its excitation energy lowers with increasing mass. On the other hand, little is still known for medium-mass nuclei. Unstable oxygen isotopes, for example, show a behavior more similar to the NCSM results of Fig. 5, i.e., a broad dipole response spreading continuously from the giant resonance region down to the PDR [8]. The proton PDR, if discovered, could also have a different qualitative behavior, as the Coulomb repulsion affects its excitation energy. The shell model predicts a centroid of the PDR of $\approx 11.6 \mathrm{MeV}$ for ${ }^{34} \mathrm{Ar}$ (see Table II) which may appear particularly large. However, this could be understood in view of the expected Coulomb effect. The large centroid might also be due to a shortcoming of the $V_{\mathrm{UCOM}}$ interaction: calculations 
based on this force have reported too small radii [25] and correspondingly they overestimate the energy of GDRs [34] at the RPA level. Although recent second RPA calculations which include $2 \mathrm{p} 2 \mathrm{~h}$ configurations seem to cure this issue [35]. The inclusion of three-nucleon forces may also play a role. Planned experiments, aimed at observing low-energy dipole response of proton-rich Ar isotopes [36], will help in testing the accuracy of the present approach. If the pygmy states in ${ }^{34} \mathrm{Ar}$ are actually observed within a few MeVs of the IVGDR tail, a substantial mixing should be expected. This would result in a broad PDR, seen as an extended low-energy tail of the isovector dipole distribution.

Finally, we note that pairing effects in the final states are partially included in the largest model spaces considered here.
A comparison of the last two rows in Table II shows that these do not generate significant changes. Nevertheless, some effects could still come including higher configurations. Including up to $1 \mathrm{p} 1 \mathrm{~h}$ excitations in the initial state effectively limits the present calculations to assuming a spherical ground state for both ${ }^{32} \mathrm{Ar}$ and ${ }^{34} \mathrm{Ar}$. However, an estimate of the quadrupole transitions based on the universal sd (USD) interaction [37] does not suggest strong deformations for these isotopes.

\section{ACKNOWLEDGMENTS}

We thank K. Boretzky, T. Aumann, R. Roth, and S. Bacca for several useful discussions.
[1] S. Goriely and E. Khan, Nucl. Phys. A706, 217 (2002).

[2] S. Goriely, E. Khan, and M. Samyn, Nucl. Phys. A739, 331 (2004).

[3] J. Piekarewicz, Phys. Rev. C 73, 044325 (2006).

[4] N. Ryezayeva et al., Phys. Rev. Lett. 89, 272502 (2002).

[5] J. Enders et al., Nucl. Phys. A724, 243 (2003).

[6] T. Hartmann, M. Babilon, S. Kamerdzhiev, E. Litvinova, D. Savran, S. Volz, and A. Zilges, Phys. Rev. Lett. 93, 192501 (2004).

[7] U. Kneissl, N. Pietralla, and A. Zilges, J. Phys. G: Nucl. Part. Phys. 32, R217 (2006).

[8] A. Leistenschneider et al., Phys. Rev. Lett. 86, 5442 (2001).

[9] P. Adrich et al., Phys. Rev. Lett. 95, 132501 (2005).

[10] G. Colò and P. F. Bortignon, Nucl. Phys. A696, 427 (2001).

[11] D. Sarchi, P. F. Bortignon, and G. Colò, Phys. Lett. B601, 27 (2004).

[12] N. Tsoneva, H. Lenske, and Ch. Stoyanov, Nucl. Phys. A731, 273 (2004).

[13] N. Tsoneva, H. Lenske, and Ch. Stoyanov, Phys. Lett. B586, 213 (2004).

[14] N. Tsoneva and H. Lenske, arXiv:0706.4204v1 [nucl-th].

[15] N. Paar, P. Ring, T. Nikšić, and D. Vretenar, Phys. Rev. C 67, 034312 (2003).

[16] N. Paar, D. Vretenar, and P. Ring, Phys. Rev. Lett. 94, 182501 (2005).

[17] E. Litvinova, P. Ring, and D. Vretenar, Phys. Lett. B647, 111 (2007).

[18] R. Roth, T. Neff, H. Hergert, and H. Feldmeier, Nucl. Phys. A745, 3 (2004), and references therein.
[19] R. B. Wiringa, V. G. J. Stoks, and R. Schiavilla, Phys. Rev. C 51, 38 (1995).

[20] A. L. Fetter and J. D. Walecka, Quantum Theory of ManyParticle Physics (McGraw-Hill, New York, 1971).

[21] W. H. Dickhoff and D. Van Neck, Many-Body Theory Exposed! (World Scientific, Singapore, 2005).

[22] G. Baym and L. P. Kadanoff, Phys. Rev. 124, 287 (1961).

[23] G. Baym, Phys. Rev. 127, 1391 (1962).

[24] R. Roth, H. Hergert, P. Papakonstantinou, T. Neff, and H. Feldmeier, Phys. Rev. C 72, 034002 (2005).

[25] R. Roth, P. Papakonstantinou, N. Paar, H. Hergert, T. Neff, and H. Feldmeier, Phys. Rev. C 73, 044312 (2006).

[26] C. Barbieri, N. Paar, R. Roth, and P. Papakostantinou, arXiv:nucl-th/0608011v1 (2006).

[27] P. Navrátil and W. E. Ormand, Phys. Rev. C 68, 034305 (2003).

[28] E. Caurier and F. Nowacki, Acta Phys. Pol. 30, 705 (1999).

[29] Shell model code ANTOINE, http://sbgat194.in2p3.fr/ theory/ antoine/menu.html.

[30] B. L. Berman and S. C. Fultz, Rev. Mod. Phys. 47, 713 (1975).

[31] D. Gazit, N. Barnea, S. Bacca, W. Leidemann, and G. Orlandini, Phys. Rev. C 74, 061001(R) (2006).

[32] S. Bacca, Phys. Rev. C 75, 044001 (2007).

[33] Table of Isotopes, 8th ed., edited by R. Firestone and V. Shirley (Wiley, New York, 1996).

[34] N. Paar, P. Papakonstantinou, H. Hergert, and R. Roth, Phys. Rev. C 74, 014318 (2006).

[35] P. Papakonstantinou and R. Roth, arXiv:0709.3167v1 [nucl-th] (2007).

[36] K. Boretzky et al. (private communication).

[37] E. K. Warburton and B. A. Brown, Phys. Rev. C 46, 923 (1992). 